\title{
Campylobacter coli
}

National Cancer Institute

\section{Source}

National Cancer Institute. Campylobacter coli. NCI Thesaurus. Code C86230.

A species of microaerophilic, Gram-negative, curved or spiral bacilli assigned to the phylum Proteobacteria. This species is motile with a single flag ellum at each pole, nonspore forming, oxidase positive, and does not hydrolyze hippurate. C. coli is commensal in pigs, associated with indigenously acquired food borne disease, and recognized as the second most common pathogen for human campylobacteriosis. 